Group for their support and dedication in providing data and advice. The many general practitioners and family practitioner committees who now have responsibility for some of the patients were a resource without which the follow up would not have been possible. The chief scientist office of the Scottish Home and Health Department has supported the project enthusiastically for several years.

1 Blumberg HH. British users of opiate-twpe drugs: a follow up study, $B r f$ Addict 1976:71:65-77.

2 Stimson GV, Oppenheimer E, Thorley A. Seven-year follow-up of heroin addicts: drug use and current outcome. Br Med f 1978:i:1190-2

Vaillant G. A 20-year follow-up of New York narcotic addicts, Arch Gen Psychiatry 1973:29:237-41.

4 Chapple PAL, Somekh DE, Taylor ME. Follow-up of cases of opiate addiction from the time of notification to the Home Office. Br Med f 1972;ii:680-3.

5 Wille R. Ten year follow-up of a representative sample of London heroin addicts, clinic attendance, abstinence and mortality. Br $\mathcal{F}$ Addict 1981; 76:259-66.

6 Bewley TH, Ben-Arie $\mathrm{O}$. Morbidity and mortality from heroin dependence: study of 100 consecutive in-patients Br Med J 1968;i:727-30.

Ghodse AH, Sheenan M, Taylor C, Edwards G. Deaths of drug addicts in the United Kingdom 1967-1981. Br Med f 1985;290:425-8.
8 Winick C. Maturing out of narcotic addiction. Bulletin of Nurcotics 1962:14

9 Marlat GA, George WH. Relapes prevention and overview of the model. $\mathrm{Br} y$ ddict 1984;79:261-73.

10 Robertson JR, Bucknall ABV, Skidmore CA, et al. Remission and relapse in heroin users and implications for management: treatment contol or risk reduction. Int $\mathcal{F}$ Addict 1989;24:229-46.

11 Department of Health and Social Security. AIDS and drug misuse part l: report of the Advisory Council on the Misuse of Drugs. London: HMSO, 1988.

12 Department of Health. AIDS and drug misuse part 2: report of the Adrison Council on the Misuse of Drugs. London: HMSO, 1989.

13 Roberts JJK, Skidmore CA, Robertson JR. Human immunodeficiency virus in drug users and increased consultation in General Practice. I R Coll Gen Pract 1989:39:373-4.

14 Robertson JR, Bucknall ABV, Welsby PD, et al. Epidemic of AIDS related virus ( $\mathrm{H} T \mathrm{LV}$-III/LAV) infection among intravenous drug abusers. Br.Med 1986:292:527-9.

15 Stimson GV, Oppenheimer E. Heroin addiction: treatment and control in Britain. London: Tavistock, 1982

16 Robertson JR. Heroin, AIDS and society. London: Hodder and Stoughton, 1987.

17 Robertson JR, Skidmore CA. AIDS in the family: the second report of th Edinburgh drug addiction study. Edinburgh: Scottish Home and Health Department, 1989.

(Accepted l December 1989)

\title{
Making better decisions: construction of clinical scoring systems by the Spiegelhalter-Knill-Jones approach
}

\author{
D G Seymour, M Green, F G Vaz
}

\begin{abstract}
A study was carried out assessing the practical use of a simple system of scoring information which can help in making a diagnosis or establishing a prognosis in an individual patient. The system was introduced in 1984 for use in gastroenterology, but it can be employed in a wide range of medical and surgical settings. This series was concerned with predicting postoperative respiratory complications in a group of elderly surgical patients.
\end{abstract}

The system combines elements from Bayes's theorem and logistic regression, though no mathematical knowledge is required to apply it in clinical practice. The method by which results are presented is easy to understand, yet at the same time more complex ideas such as conflict of evidence and doubt may be embraced if the clinician so desires.

\section{Introduction}

Weighing up signs, symptoms, and test results in an individual patient, comparing them with past experience, and arriving at a reasoned decision lie at the heart of clinical medicine. Recently Spiegelhalter and Knill-Jones have proposed a simple scoring system which can add precision to risk assessment in individual patients. ${ }^{1-4}$ To the non-mathematical user the great attraction of the system is that it demands only the ability to add, subtract, and look up a reference table or graph. Despite its simplicity, however, the system has a firm statistical basis and can handle complex diagnostic concepts such as conflict of evidence and doubt. ${ }^{1}$ The system can be used equally well to establish prognosis or to estimate the likelihood of a particular diagnosis (as in the original application). ${ }^{\prime}$ This paper examines the system in relation to establishing prognosis.

The Spiegelhalter-Knill-Jones approach has been welcomed by many statisticians (see discussion after Spiegelhalter and Knill-Jones') because of the elegance with which it combines elements of Bayes's theorem with those of logistic regression. The result is a system which neatly sidesteps some of the main disadvantages of the two original techniques. For example, it does not assume that all risk factors are acting independently within each outcome class (the "independence Bayes" assumption, which is central to many bayesian analyses), while at the same time predictions are presented in a form which is less mathematical and much more clinically relevant than the output of conventional logistic regression analysis.

We believe that the Spiegelhalter-Knill-Jones system deserves to be better known in clinical medicine, and this paper therefore focuses on the practicalities of using the technique rather than on its statistical origins. We refer to these, however, in the appendix and discussion.

\section{Present study and results}

SOURCE OF ILLUSTRATIVE DATA

All patients who served in the assessment were aged 65 or over and received a general surgical operation. They comprised 258 patients from Dundee (age range 65-93) and 299 patients from Cardiff (age range 65-97). Two thirds of the patients in both data sets were aged between 65 and 74 . Full details of the patients are given elsewhere.

Many variables relating to preoperative state and postoperative outcome were collected prospectively in both data sets by using criteria established in advance and which are described elsewhere. ${ }^{67}$ As our purpose is to illustrate the Spiegelhalter-Knill-Jones approach with a simple clinical example, this paper looks simply at the ability of four preoperative risk factors (preoperative chest disease, smoking in the six weeks before admission, site of the surgical incision, and presence of volume depletion) to predict postoperative respiratory complications. Full details of how variables were defined in the larger study and of how these four respiratory variables were selected from an initial list of 19 have been reported. ${ }^{5-7}$ Brief definitions are given in table I.

The collection of two data sets, the first from Dundee and the second from Cardiff, was deliberate. Data collection from both data sets was completed before any calculations were performed, but the weightings on which the Spiegelhalter-Knill-Jones system is based were calculated solely from the patients from Dundee, who thus made up the "training" data set. The weightings obtained from the training data set were then applied to the Cardiff group - that is, the
Correspondence to: Dr Seymour. 
"test" data set. This procedure was carried out because the acid test of any predictive system is not how it performs in the training data set from which it was derived but the quality of its predictions in another, test data set.

\section{CALCULATION OF SPIEGELHALTER-KNILL-JONES} WEIGHTINGS

Once the Spiegelhalter-Knill-Jones weightings have been derived they can be used by the nonmathematician, but the process of derivation from the training data set requires statistical skill (see appendix). The fruits of these statistical calculations (which are all the non-mathematical user needs when applying the system) are "adjusted weights of evidence" for each of the predictor variables together with a "starting score," which is constant for a given outcome variable. In the example used in this assessment the outcome variable was a postoperative respiratory complication and the starting score was derived from the incidence of this problem in the training data set. (In broad terms the starting score can be thought of as reflecting the average risk of postoperative respiratory complications in elderly general surgical patients, and the weights of evidence may be viewed as tools for refining the risk prediction by taking into account key preoperative findings in the individual patient.)

\section{APPLYING SPIEGELHALTER-KNILL-JONES WEIGHTINGS TO INDIVIDUAL PATIENTS}

Table I gives the starting score and adjusted weights of evidence needed to predict the chances of a postoperative respiratory complication from the four

TABLE I-Prediction of postoperative respiratory complications by using four preoperative variables scored by Spiegelhalter-Knill-fones system *

\begin{tabular}{|c|c|c|}
\hline & & $\begin{array}{l}\text { Spiegelhalter- } \\
\text { Knill-Jones score }\end{array}$ \\
\hline \multicolumn{2}{|c|}{ Starting score (all patients) } & -41 \\
\hline \multicolumn{3}{|c|}{ Adjusted wetghts of evrdence } \\
\hline \multicolumn{2}{|c|}{ Preoperative chest problems $\dagger\left\{\begin{array}{l}\text { Yes } \\
\text { No }\end{array}\right.$} & $\begin{array}{l}+85 \\
-38\end{array}$ \\
\hline \multicolumn{2}{|c|}{ Smoking in past six weeks $\begin{array}{l}\text { Yes } \\
\text { No }\end{array}$} & $\begin{array}{l}+51 \\
-33\end{array}$ \\
\hline Incision site & $\begin{array}{l}\text { Upper abdomen or thorax } \\
\text { Mid-abdomen or lower abdomen } \\
\text { Hernia or non-abdominal }\end{array}$ & $\begin{array}{l}+47 \\
-7 \\
-54\end{array}$ \\
\hline \multicolumn{2}{|c|}{ Volume depletion $\left\{\begin{array}{l}\text { Moderate or severe } \neq \\
\text { Nil or minor }\end{array}\right.$} & $\begin{array}{r}+234 \\
-13\end{array}$ \\
\hline
\end{tabular}

*Postoperative respiratory complication taken as two or more of the following: cough productive of sputum; temperature $\geqslant 38^{\circ} \mathrm{C}$; physical signs (coarse crepitations, bronchial breathing, poor air entry) not present before operation.

†Preoperative chest problem taken as history of chronic bronchitis (cough and sputum for more than three months of the year for over two years), or chronic or recurrent chest disease requiring repeated treatment, or respiratory signs elicited by investigator.

$\ddagger$ Volume depletion graded as moderate or severe if investigator judged that two or more of the following were present that were not caused by other factors such as cardiac disease or sepsis: postural hypotension $(\geqslant 20 \mathrm{~mm} \mathrm{Hg})$; factors such as cardiac disease or sepsis: postural hypotension $(\geqslant 20 \mathrm{~mm} \mathrm{Hg})$;
systolic blood pressure < $110 \mathrm{~mm} \mathrm{Hg}$; pulse $>100$ beats $/ \mathrm{min}$; absence of systolic blood pressure $<110 \mathrm{~mm} \mathrm{Hg}$; pulse $>100$ beats $/ \mathrm{min}$; absence of
signs of fluid overload; cutaneous lividity; oliguria or rise in blood urea signs of fluid overload; cutaneous lividity; oliguria or rise in blood urea
concentration from non-renal cause; history consistent with volume depletion-for example, vomiting.

TABLE II -Obtaining percentage prediction from total score $\left(T^{\star}\right)$

\begin{tabular}{lc||cc}
\hline$T^{\star}$ & \% Probability of predicted outcome & $T^{\star}$ & \% Probability of predicted outcome \\
\hline- Infinity & 0 & +20 & 55 \\
-294 & 5 & +41 & 60 \\
-220 & 10 & +62 & 65 \\
-173 & 15 & +85 & 70 \\
-139 & 20 & +110 & 75 \\
-110 & 25 & +139 & 80 \\
-85 & 30 & +173 & 85 \\
-62 & 35 & +220 & 90 \\
-41 & 40 & +294 & 95 \\
-20 & 45 & + Infinity & 100 \\
0 & 50 & & \\
\hline
\end{tabular}

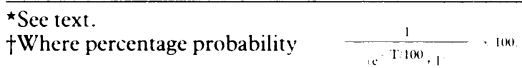

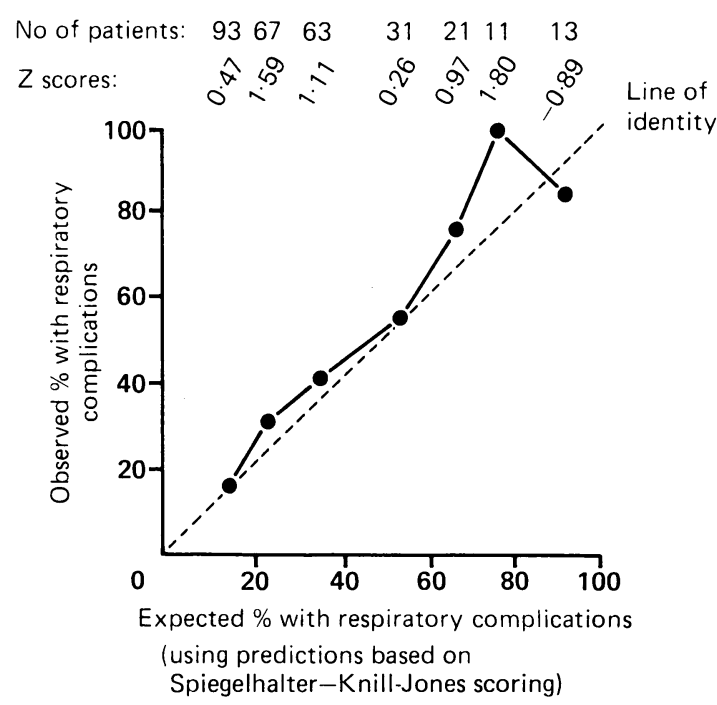

FIG 1-Observed and expected (predicted) rates of postoperative respiratory complications in test data set 299 patients aged 65 and over from Cardiff). Perfect predictor would coincide exactly with diagonal running from bottom left to top right (line of identity). As values of $Z$ statistic lie within range -2 to +2 it is inferred that fit of predictor to line of identity is satisfactory. ${ }^{2}$ Predictions are therefore "reliable"

preoperative risk factors. To predict the risk of postoperative respiratory problems in an individual patient two steps are required. Firstly, by referring to table I add the starting score to the appropriate adjusted weights of evidence for that patient. Then, by referring to table II (or using the formula in the appendix) convert the total score obtained to a percentage risk. These steps are illustrated in the following example.

Hypothetical case-The patient has a history of chronic bronchitis, does not currently smoke, is having upper abdominal surgery, and has no evidence of preoperative volume depletion. Step 1 (table I): add the starting score $(-41$ in all cases) to the adjusted weights of evidence for preoperative chest disease $(+85)$, non-smoking $(-33)$, upper abdominal incision $(+47)$, and absence of volume depletion $(-13)$. The total score is +45 (this is referred to as "T" by Spiegelhalter ${ }^{2}$ and is the convention used in table II). Step 2 (table II): convert the total score (T) of +45 into a percentage prediction. A $\mathrm{T}$ of +45 corresponds to a predicted probability of postoperative respiratory complications of between $60 \%$ and $65 \%$.

\section{HOW ACCURATE ARE THE PREDICTIONS?}

As stated above, the key test of any system of prediction is not how it performs in the training data set from which it was derived but whether it retains its predictive value in another (test) data set. Numerous elaborate methods are available to examine predictive accuracy, ${ }^{2}$ but we present two simple pictorial methods. Figure 1 shows the first method. The percentage of postoperative respiratory complications observed in patients in the test data set is plotted against the percentage of postoperative respiratory complications which would have been expected if the calculated predictions had been perfect. The line produced is close to the diagonal (line of identity) running from bottom left to top right, the diagonal representing perfect correspondence between predicted and actual outcomes. The result indicates that the predicted probabilities are "reliable" - that is, patients predicted to have, say, $60 \%$ chance of a complication will actually develop that complication around $60 \%$ of the time. Table III gives the data from which fig 1 is constructed.

The second method of examining the quality of prediction is to use receiver operating characteristic curves which simultaneously look at the sensitivity and specificity of prediction when using a range of thres- 
TABLE III-Postoperative respiratory complications in elderly general surgical patients in Dundee and Cardiff: comparison of observed outcome with that predicted by Spiegelhalter-Knill-fones system *

\begin{tabular}{|c|c|c|c|}
\hline \multicolumn{2}{|r|}{ Predicted outcome } & \multicolumn{2}{|c|}{$\begin{array}{l}\text { Observed outcome } \\
\text { (No }(\%) \text { of patients) }\end{array}$} \\
\hline $\mathrm{T} \dagger$ & $\begin{array}{l}\% \text { Predicted to have postoperative } \\
\text { respiratory complications }\end{array}$ & $\begin{array}{c}\text { Postoperative respiratory } \\
\text { complications in Dundee } \\
\text { data set }\end{array}$ & $\begin{array}{c}\text { Postoperative respiratory } \\
\text { complications in Cardiff } \\
\text { data set }\end{array}$ \\
\hline$<-221$ & 0 to $9 \cdot 9$ & $0 / 0$ & $0 / 0$ \\
\hline-220 to -140 & 10 to $19 \cdot 9$ & $4 / 37(10 \cdot 8)$ & $15 / 93(16 \cdot 1)$ \\
\hline-139 to -86 & 20 to $29 \cdot 9$ & $14 / 61(23 \cdot 0)$ & $21 / 67(31 \cdot 3)$ \\
\hline-85 to -42 & 30 to $39 \cdot 9$ & $26 / 68(38 \cdot 2)$ & $26 / 63(41 \cdot 3)$ \\
\hline-41 to -40 & 40 to $59 \cdot 9$ & $22 / 41(53.7)$ & $17 / 31(54.8)$ \\
\hline+41 to -84 & 60 to $69 \cdot 9$ & $16 / 25(64 \cdot 0)$ & $16 / 21(76 \cdot 2)$ \\
\hline+85 to -138 & 70 to $79 \cdot 9$ & $11 / 17(64 \cdot 7)$ & $11 / 11(100 \cdot 0)$ \\
\hline$>+139$ & 80 to $100 \cdot 0$ & $9 / 9(100.0)$ & $11 / 13(84 \cdot 6)$ \\
\hline
\end{tabular}

$\star$ Dundee patients $=$ training data set $(n=258)$. Cardiff patients $=$ test data set $(n=299)$.

†Total score. See text and table II former system is preferable because of the clarity with which it presents its results. In addition, we (like others' $\left.{ }^{\prime}\right)$ find that the Spiegelhalter-Knill-Jones system produces predictions which are more reliablethat is, less overconfident - than those provided by an independence Bayes approach, ${ }^{5}$ though in simple examples such as that given above the predictions produced by the two approaches may differ by only a few percentage points. (In the terminology used in the appendix an independence Bayes approach is equivalent to using the basic weights of evidence rather than the adjusted weights of evidence.)

\section{Discussion}

Over the past two decades three numerical approaches have been prominent in clinical decision making, ${ }^{\prime}$ and all have their strengths and weaknesses. The independence Bayes method is based on classic statistical techniques and when suitably presented 1011 is often highly acceptable to clinicians. Unfortunately, its underlying assumption (that risk factors in the predictive equation act independently of one another within outcome categories) may lead to overconfident estimates when several risk factors are contributing to the prediction. ${ }^{23}$ Careful selection of risk factors which are not strongly correlated with one another may get over some of the problems, and advocates of the independence Bayes approach point to its robustness in practical clinical settings." Other classic statistical techniques such as logistic regression and discriminant function analysis are mathematically sounder when the interplay among several risk factors is being considered, but they tend to present their predictions in a format that is alien to many clinicians. ${ }^{12}$ Expert systems are based on diagnostic rules rather than classical statistical techniques. As such they tend to make their predictions in a format that is familiar to clinicians, but as a radical departure from classical statistical techniques these systems have been viewed with concern by many statisticians (see discussion after Spiegelhalter and Knill-Jones $^{1}$ ). Another practical objection is that expert systems may require elaborate and expensive computer software and are often difficult to evaluate.

Spiegelhalter-Knill-fones method compared with independence Bayes and logistic regression-The Spiegelhalter-Knill-Jones method combines elements of independence Bayes and logistic regression but has advantages over both these methods. It is usually more accurate than independence Bayes ${ }^{5}$ and promises to be more clinically acceptable than logistic regression. The new system is likely to be clinically acceptable because it mirrors the way that many clinicians already make diagnoses (by weighing up points for and against) and is mathematically simple to use. Use may be simplified still further by putting the system on a microcomputer, which can prompt the questions, carry out the minimal calculations, and possibly express the results graphically. A microcomputer application of the GLADYS system for diagnosis in gastroenterology has recently been devised. ${ }^{13}{ }^{14}$ A further practical advantage of the Spiegelhalter-Knill-Jones system is that variables whose values are missing in a given patient may be weighted as zero, a much simpler technique than that required to deal with missing values in regression equations.

Spiegelhalter-Knill-fones method and knowledge based concepts-Under the usual terminology the Spiegelhalter-Knill-Jones method (like logistic regression) is a "probabilistic" approach, as opposed to the "knowledge based" approach of artificial intelligence, of which "expert systems" are an example. In the past the differences between the two types of approach have often been emphasised and much has been made of the ability of knowledge based systems to 
explain their reasoning and to cope with concepts such as doubt and conflict of evidence. Though the Spiegelhalter-Knill-Jones system is firmly probabilistic, its "points for and points against" approach clearly indicates the reasoning behind a prediction and can be used to give numerical estimates of doubt and conflict of evidence. 'It therefore builds a bridge between the probabilistic and knowledge based schools and reflects an encouraging trend to bring together groups of workers who have not always been keen to explore alternative methods of diagnosis and prognosis.

Numerical techniques as upholders of traditional clinical method-The application of numerical or computer techniques, or both, to clinical diagnosis is likely to produce a renaissance of interest in traditional clinical skills. ${ }^{16-18}$ Thus in order to enter clinical information into a computer it is first necessary for signs and symptoms to be defined rigorously. Once the process of analysis begins it may become obvious that certain signs and symptoms are much more important than others. For example, table I shows that the presence of volume depletion is more strongly associated with postoperative respiratory complications than any of the other variables examined. Unfortunately, volume depletion is also the most difficult of the variables to define, suggesting that future studies should consider more refined means of measuring fluid and electrolyte disturbance. This type of interplay between clinical and numerical methods can be very rewarding. Arguably the greatest benefit produced by the Spiegelhalter-Knill-Jones method and similar techniques is the new life breathed into the traditional clinical skills of history taking, careful physical examination, and judicious selection of diagnostic tests.

We are grateful to Dr David Spiegelhalter, Dr Robin KnillJones, and Dr Ted Coles for helpful comments on the manuscript, and to Research into Ageing (formerly the British Foundation for Age Research) for financial support.

\section{Appendix}

Summary of statistical techniques used to derive SpiegelhalterKnill-fones weightings

I. The independence Bayes equation may be expressed as ${ }^{10}$ 10: $^{10}$ :

Posterior odds $=$ prior odds $\times$ LR of variable $1 \times$

$\mathrm{LR}$ of variable $2 \ldots \times \mathrm{LR}$ of variable $\mathrm{N}$

where posterior odds are the predicted odds of the outcome in an individual, prior odds are the odds of the outcome in the population under study, and LR= likelihood ratio. To calculate the likelihood ratio for a particular value of a variable the patients are divided into those who develop a given outcome and those who do not, and the frequency of the particular value of the variable in these two groups is compared. Where the variable is binary the following equation may be used:

Likelihood ratio $=($ sensitivity $) /(1-$ specificity $)$

for a positive test result; or likelihood ratio $=$

$(1-$ sensitivity $) /($ specificity) for a negative test result.

A continuity correction for the likelihood ratio is advisable (see Spiegelhalter').

II. Taking natural logarithms and multiplying by 100 , equation (1) becomes:

$100 \log _{\mathrm{e}}$ posterior odds $=100 \log _{\mathrm{e}}$ prior odds +

$100 \log _{\text {. }}$ LR of variable $1+100 \log$. LR of

variable $2 \ldots+100 \log _{c} L R$ of variable $N$.

$$
\text { (2) }
$$

III. By using the terminology of the Spiegelhalter-KnillJones method, equation (2) becomes:
Total score $(\mathbf{T})=$ starting score + weight of evidence of variable $1+$ weight of evidence of variable $2 \ldots+$ weight of evidence of variable $\mathrm{N}$.

IV. Equation (3) assumes that the variables are independent of one another (within each outcome class), which is unrealistic in many clinical settings. To allow for this the Spiegelhalter-Knill-Jones method calculates "adjusted weights of evidence," which are obtained by entering the raw weights of evidence as independent variables in a logistic regression equation.' This is conveniently done by the generalised linear interactive modelling statistical package.

This produces the predictive equation in its final form:

Total score $(T)=$ starting score + adjusted weight of evidence for variable 1 +adjusted weight of evidence for variable $2 \ldots$. +adjusted weight of evidence for variable $\mathrm{N}$.

V. Because $T=100 \log _{\mathrm{e}}$ posterior odds and because odds= $(\mathrm{p} / \mathrm{l}-\mathrm{p})$ it may be calculated that:

Predicted probability $($ in $\%)=$

$$
\frac{\mathrm{e}^{\mathrm{T} /(10)}}{1+\mathrm{e}^{\mathrm{T}(100)}} \times 100=\frac{1}{\mathrm{e}^{\mathrm{T}(1000}+1} \times 100 .
$$

For full specifications of weightings see Spiegelhalter and

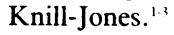

1 Spiegelhalter DJ, Knill-Jones RP. Statistical and knowledge-based approaches oclinical decision-support systems, with an application in gastroenterology. Journal of the Royal Statistical Society A 1984;147:35-77.

2 Spiegelhalter DJ. Statistical methodology for evaluating gastrointestinal symptoms. Clinics in Gastroenterology 1985:14:489-515.

3 Spiegelhalter DJ. Probabilistic prediction in patient management and clinical trials. Stat Med 1986;5:421-33.

4 Knill-Jones RP. Diagnostic systems as an aid to clinical decision making. BrMed f 1987;295:1392-6.

5 Seymour DG. Prediction of risk in the elderly surgical patient. Birmingham: University of Birmingham, 1988. (MD thesis.)

$6 \mathrm{Vaz} \mathrm{FG}$, Seymour DG. A prospective study of elderly general surgical patients. I. Pre-operative medical problems. Age Ageing 1989;18:309-15.

7 Seymour DG, Vaz FG. A prospective study of elderly general surgical patients. II. Post-operative complications. Age Ageing 1989;18:316-26.

8 Wasson JH, Sox HC, Neff RK, Goldman L. Clinical prediction rules. Applications and methodological standards. N Engl F Med 1985;313:793-9. 9 Hanley JA, McNeil BJ. The meaning and use of the area under a receiver operating characteristic (ROC) curve. Radiology 1982;143:29-36.

10 Simel DL. Plaving the odds. Lancet 1985;i:329-30 (plus correction p 940).

11 Adams ID, Chan M, Clifford PC, et al. Computer aided diagnosis of acute abdominal pain: a multivariate study. Br Med f 1986;293:800-4.

2 Seymour DG. Medical assessment of the elderly surgical patient. London: Croom Helm, 1986.

13 Knill-Jones RP. A computer-assisted diagnostic system for dyspepsia (GLADYS). In: Tsiftis DD, ed. Lecture notes in medical informatics 28. Proceedings of a workshop on objective medical decision making, system approach Proceedings of a workshop on objective medical decision making, sys
and disease, Crete, 1985. Berlin: Springer Verlag, 1986:215-26.

14 Card WI, Lucas RW. Computer interrogation in medical practice. International fournal of Man-Machine Studies 1981;14:49-57.

15 Spiegelhalter DJ. A statistical view of uncertainty in expert systems. In: Gale W, ed. Artificial intelligence and statistics. Reading, Massachusetts: Addison Wesley, 1986:17-55.

16 de Dombal FT. Analysis of symptoms in the acute abdomen. Clinics in Gastroenterology 1985;14:531-43.

17 Knill-Jones RP. A formal approach to symptoms in dyspepsia. Clinics in Gastroenterology 1985;14:517-29.

18 Sackett DL, Haynes RB, Tugwell P. Clinical epidemiologv. A basic science for clinical medicine. Boston: Little, Brown, 1985.

(Accepted 9 November 1989)

\section{Correction}

\section{Why are the Japanese living longer?}

A printers' error occurred in table I of this paper by Professor M G Marmot and Dr George Davey Smith (23-30 December, p 1547). The figures for life expectancy of Japanese and English and Welsh males are those at age 65 and not 85 as published.

\section{Snoring in children: association with respiratory} symptoms and passive smoking

One editorial error and one authors' error occurred in the paper by Dr Giuseppe M Corbo and others (16 December, p 1491). The 11 th line of the results section of the abstract should read never smoking households and not moderate smoking households as published and the ninth line of p 1493 should read $8 \cdot 3 \%$ and not $8 \cdot 6 \%$. 